\title{
Complete Patient Form
}

National Cancer Institute

\section{Source}

National Cancer Institute. Complete Patient Form. NCI Thesaurus. Code C128508.

The healthcare provider, healthcare setting, and/or patient must complete a form such as a patient agreement or patient-prescriber agreement form. 